\title{
Civilisations
}

Revue internationale d'anthropologie et de sciences

humaines

42-1 | 1993

Amérique latine-Europe

\section{Cuba - Europe : rencontres et reculs}

\section{Eduardo Perera Gomez}

\section{OpenEdition \\ Journals}

Édition électronique

URL : http://journals.openedition.org/civilisations/2115

DOI : 10.4000/civilisations.2115

ISSN : 2032-0442

\section{Éditeur}

Institut de sociologie de l'Université Libre de Bruxelles

\section{Édition imprimée}

Date de publication : 1 novembre 1993

Pagination : 237-241

ISBN : 0009-8140

ISSN : 0009-8140

\section{Référence électronique}

Eduardo Perera Gomez, «Cuba - Europe : rencontres et reculs », Civilisations [En ligne], 42-1 | 1993, mis en ligne le 30 novembre 1996, consulté le 15 septembre 2020. URL : http:// journals.openedition.org/civilisations/2115

Ce document a été généré automatiquement le 15 septembre 2020

(C) Tous droits réservés 


\title{
Cuba - Europe : rencontres et reculs
}

\author{
Eduardo Perera Gomez
}

\section{NOTE DE L'ÉDITEUR}

Ce texte est une version de la communication présentée par l'auteur au séminaire «Cuba : la révolution isolée? », Fritz-Erler-Akademie, Freudenstadt, RFA, décembre 1991.

1 Pour aborder les relations entre l'Europe et Cuba, il faut considérer trois éléments essentiels : l'appartenance de Cuba à la scène latino-américaine, avec tous les facteurs à résonance géopolitique qui en découlent; le choc fréquent entre volontés et réalités politiques ; et enfin - mais ce n'est pas le moins important - le caractère de ce que l'on appelle « l'isolement » cubain, dérivé essentiellement d'actions extérieures conscientes.

\section{Le contexte régional et sa résonance géopolitique}

2 Les relations de l'Europe avec Cuba s'insèrent dans le cadre général des relations de l'Ancien continent avec l'Amérique latine, qui n'était pas - et n'est pas - pour les Européens une priorité dans le Tiers-Monde. Cette position influe sur l'existence de certaines conditions communes qui, en modelant le schéma global des liens interrégionaux, se manifestent aussi dans le cas cubain.

3 En termes de sphères d'influence, la région se situe de toute évidence en dehors de la zone traditionnelle d'action européenne, pour des raisons historiques aussi bien que géopolitiques, qui découlent de la présence régionale des Etats-Unis, rehaussée par l'alliance stratégique entre ceux-ci et les pays européens membres de l'OTAN. Mais bien que l'Europe soit entrée dans des contradictions conjoncturelles avec Washington, la marge de manoeuvre dont elle a pu disposer dans ses relations avec l'Amérique latine est restée assez étroite dans l'ensemble, que ce soit par désintérêt spécifique, par autolimitation, ou comme résultante directe de ses liens particuliers avec les EtatsUnis. A cela s'ajoute le fait que, du côté latino-américain, des inconvenances ont réduit 
la possibilité d'identifier clairement un interlocuteur valable, ayant un pouvoir de négociation suffisant pour rendre cohérent, si pas le sous-continent tout entier, du moins une bonne partie de celui-ci.

4 Dans le cas de Cuba, un élément spécifique réside dans la différence de système socioéconomique, qui a considérablement pesé sur les relations mutuelles durant la guerre froide, provoquant bon nombre d'impressions fausses, de malentendus et d'hésitations dans les relations de part et d'autre. Cuba était traditionnellement identifié par les chancelleries européennes comme un pays « de l'Est», bien plus que comme un pays latino-américain; un tel point de vue ne peut être considéré indépendamment de ce qu'étaient les engagements cubains de l'époque, ni de l'optique résolument « de bloc » qui dominait dans les relations internationales. Ainsi, un facteur important de la spécificité des relations entre, Cuba et la Communauté a, durant de nombreuses années, découlé de l'adhésion de Cuba au Conseil d'Aide Economique Mutuelle (CAME), étant donné la non reconnaissance mutuelle entre celui-ci et la Communauté européenne.

\section{Volonté US réalités politiques}

5 Dans le domaine des relations entre Cuba et l'Europe, la réalité a, en de nombreuses occasions, dépassé les volontés politiques. Pour une bonne part, cela explique les causes, les obstacles et /ou les limitations caractérisant les relations entre Cuba et l'Europe occidentale qui ont conduit aux principales situations de recul. En voici quelques-unes.

Cuba s'est vu contraint à une relation économique très étroite avec l'Union Soviétique et le reste des anciens pays socialistes européens. Mais même dans de telles conditions, il n'a pas renoncé à ses liens avec l'Ouest européen, atteignant à certains moments un commerce d'importation qui dépendait pour plus de $20 \%$ de sources non socialistes, dont plus de la moitié était réalisée avec l'Europe occidentale, et une grande partie avec les pays de la Communauté économique européenne.

7 Et pourtant, en termes réels, les relations commerciales cubaines avec l'Europe occidentale n'ont habituellement pas dépassé les 8 à $9 \%$ du total du commerce de Cuba. Ce chiffre ne constitue pas un sous-produit des étroites relations de Cuba avec les anciens pays socialistes européens, mais bien l'un des résultats tangibles de la dépendance cubaine du marché nord-américain avant 1959. Les causes remontent à la deuxième moitié du XIX ${ }^{e}$ siècle, au moment où les Etats-Unis ont supplanté les marchés britannique et espagnol dans le commerce extérieur de Cuba; l'espace commercial restreint avec l'Europe est donc davantage un héritage que le résultat d'un processus mené consciemment.

Dans les années 60, avant son adhésion au CAME, Cuba a trouvé en Europe occidentale d'importantes solutions alternatives à ses problèmes de commerce extérieur, ce dont il ne faut pas s'étonner si l'on considère, d'une part, le caractère ouvert de l'économie cubaine et, d'autre part, de fortes différences d'attitude vis-à-vis de Cuba dans plusieurs pays de la région. On peut en donner des exemples marquants, à savoir le flux commercial existant entre l'île et la Grande-Bretagne, particulièrement de 1962 à 1965 ; les liens avec l'Espagne de Franco qui, partant d'une position différente - qui n'éliminait pas le distanciement idéologique, mais a toujours maintenu une attitude de respect vis-à-vis de la révolution cubaine - a établi un principe de troc qui a permis à Cuba d'augmenter considérablement le tonnage de sa flotte de pêche et de commerce ; 
d'importants courants d'échanges qui se sont développés avec la France et l'Italie dans le domaine des machines agricoles et du transport automoteur.

Ont participé à la formation des liens avec l'Europe occidentale tant la volonté politique déjà mentionnée, que la position de nombreux secteurs, voire de gouvernements de la région, qui ont manifesté leur opposition au blocus nord-américain, en partant d'une conception traditionnelle de rejet du mécanisme de l'embargo comme instrument de pression.

\section{Isolement voulu ? Non, isolement imposé}

10 Indépendamment de ses résultats concrets, il faut établir une nette différence entre le soi-disant «auto-isolement» de Cuba et l'isolement réel, résultat d'une politique consciemment dirigée de l'extérieur.

11 Aujourd'hui, alors qu'on souligne avec emphase la "fin de la guerre froide » et la «désidéologisation des relations internationales», la politique européenne paraît contradictoire. Non seulement elle a abandonné certaines actions pratiques d'opposition au blocus nord-américain, entreprises durant les premières années de la révolution, mais elle a aussi modifié les termes de son discours et, dans des domaines concrets, sauf exceptions, elle soutient Washington en conditionnant l'aide matérielle et financière en faveur de Cuba à la réalisation de changements internes dans le pays ${ }^{1}$.

12 Si la nécessité de modifier les termes du dialogue s'impose de part et d'autre, au niveau interrégional aussi bien que bilatéral, dans le but de dépasser les malentendus et les incompréhensions en partant d'une perception mutuelle plus réaliste, il est tout aussi vrai qu'il est incohérent de maintenir vis-à-vis de Cuba des positions et des points de vue déjà dépassés par ceux-là mêmes qui les font valoir. Il est donc indispensable, avant tout, que l'Europe fasse honneur au pluralisme qu'elle proclame et qu'elle comprenne que les politiques isolationnistes et hostiles engendrent bien souvent des effets contraires à ceux que l'on attendait.

\section{Tendances négatives actuelles}

13 L'eurocentrisme, présent dans tout le processus de formation du Marché Unique européen de 1993, et qui s'est renforcé avec les événements en Europe de l'Est et en URSS, risque bien d'exclure davantage l'Amérique latine et Cuba des priorités européennes. Cette tendance est, sans aucun doute, accentuée par le mouvement international actuel qui vise la formation de grands blocs économiques régionaux, dans laquelle domine une logique à contenu géopolitique. Il faut y ajouter les conséquences de la guerre du Golfe, parmi lesquelles l'érosion du rôle politique joué par l'Europe au niveau global, et l'unipolarité provenant de l'hégémonie politico-militaire nordaméricaine. Dans ce sens, les changements en Europe de l'Est et en URSS ont particulièrement contribué à la configuration de l'ordre international actuel et à une perte croissante des marges de manoeuvre disponibles pour les pays du Tiers-Monde dans la conduite de leurs relations extérieures, noyées à l'avenir dans la substitution progressive du conflit Est-Ouest par l'opposition Nord-Sud.

14 Toutefois, il convient de préciser que, dans le cas de Cuba, l'Europe de l'Est et l'ex-URSS ne sont pas devenues, à strictementparler, des concurrents dans le cadre des relations 
avec l'Europe occidentale, mais exactement le contraire : c'est l'Europe occidentale qui peut, et est en mesure de concurrencer Cuba dans le cadre de ces pays.

Ainsi, l'Europe de l'Est et l'actuelle communauté des Etats Indépendants (CEI), ainsi que les autres Etats nouveaux nés de l'ex-URSS, ont un espace important à couvrir dans la nouvelle architecture européenne, tout minime ou secondaire qu'il soit par comparaison avec celui qu'occupe déjà la CEE. Ceci entraîne, logiquement, des conséquences négatives pour tout le Tiers-Monde, et en particulier pour l'Amérique latine et Cuba. Parallèlement, on observe un déplacement des conflits vers le Sud sousdéveloppé, une centralisation croissante des efforts de développement et de solution de la crise dans les pays mêmes qui constituent les noyaux des projets de blocs régionaux, et une exigence accrue pour les pays du Tiers-Monde d'ajustement structurels et de libéralisation économique.

\section{Quelques perspectives}

Le thème de la non priorité de l'Amérique latine pour l'Europe présente cependant le risque d'un certain fatalisme. Si les relations avec l'Amérique latine ne sont pas essentielles pour l'Europe, ni même probablement importantes - et, dans ce cas, Cuba n'est pas non plus une exception, tenant compte du fait que, pour elle, l'essentiel est aujourd'hui sa réintégration complète sur la scène latino-américaine -, on ne peut oublier que le monde actuel est caractérisé par des relations de franche et inévitable interdépendance. Il est possible et souhaitable de développer les relations entre l'Europe, l'Amérique latine et Cuba, pour diversifier les relations extérieures mutuelles, d'alléger le poids de la dépendance spécifique de chacune de ces régions vis-à-vis des Etats-Unis, et d'atteindre un certain niveau d'équilibre dans les relations Nord-Sud afin de contribuer à sortir les pays latino-américains de la crise structurelle dans laquelle ils sont plongés. Dans ce sens, le rôle joué par les organisations non gouvernementales est un exemple important.

17 L'Europe a donc à relever le défi de valider, sans paternalismes ni ingérences, une option réelle de développement pour le sous-continent latino-américain, dans le cadre d'un processus de véritable ouverture qui, réclamé par l'Amérique latine, n'a pas de contrepartie spécifique sur le Vieux Continent.

\section{NOTES}

1. II y a toutefois des exceptions, qui se manifestent essentiellement dans le domaine commercial. L'opposition exprimée par la Communauté européenne au projet de loi Torricelli est plus cohérent avec le caractère délicat du thème du blocus pour les pays d'Europe, mais contribue aussi de manière importante à souligner la bassesse et l'illégalité d'une telle mesure. 
AUTEUR

EDUARDO PERERA GOMEZ

Centre d'études européennes, LaHavane, Cuba 